\title{
Interpreting Enthymematic Arguments Using Belief Revision
}

Journal Article

Author(s):

Brun, Georg; Rott, Hans

Publication date:

2013-12

Permanent link:

https://doi.org/10.3929/ethz-b-000075292

Rights / license:

In Copyright - Non-Commercial Use Permitted

Originally published in:

Synthese 190(18), https://doi.org/10.1007/s11229-013-0248-6 


\title{
Interpreting enthymematic arguments using belief revision
}

\author{
Georg Brun • Hans Rott
}

Received: 27 March 2012 / Accepted: 24 January 2013 / Published online: 19 February 2013

(C) Springer Science+Business Media Dordrecht 2013

\begin{abstract}
This paper is about the situation in which an author (writer or speaker) presents a deductively invalid argument, but the addressee aims at a charitable interpretation and has reason to assume that the author intends to present a valid argument. How can he go about interpreting the author's reasoning as enthymematically valid? We suggest replacing the usual find-the-missing-premise approaches by an approach based on systematic efforts to ascribe a belief state to the author against the background of which the argument has to be evaluated. The suggested procedure includes rules for recording whether the author in fact accepts or denies the premises and the conclusion, as well as tests for enthymematic validity and strategies for revising belief state ascriptions. Different degrees of interpretive charity can be exercised. This is one reason why the interpretation or reconstruction of an enthymematic argument typically does not result in a unique outcome.
\end{abstract}

Keywords Enthymeme $\cdot$ Argument $\cdot$ Interpretation $\cdot$ Charity $\cdot$ Belief revision

\section{The problem}

This paper focuses on the problem of interpreting enthymematic arguments and suggests using concepts and tools from belief revision theory. It is a case study in a belief-revision approach to interpretation.

G. Brun (凶)

ETH Zürich, CHN H 73.1, Universitätstrasse 16, 8092 Zürich, Switzerland

e-mail: Georg.Brun@env.ethz.ch

H. Rott

Department of Philosophy, Universität Regensburg, 93040 Regensburg, Germany

e-mail: hans.rott@ur.de 
Suppose that an author, i.e. a writer or speaker, has presented a deductively invalid argument with (finitely many) premises and a (single) conclusion; suppose also that there is an interpreter who has reason to assume that the author intends to present a deductively valid argument and who aims at a charitable interpretation. Our guiding question is this: How can he go about interpreting the author's reasoning as valid? ${ }^{1}$

For the sake of simplicity, we place two restrictions right at the start. Firstly, we will focus on arguments that are meant to be understood as deductively valid; that is, meant to be understood as arguments with conclusions that must be true provided all of their premises are true. ${ }^{2}$ This excludes arguments that are presented as merely supporting yet not establishing the conclusion, such as inductive arguments or arguments by analogy. Secondly, we confine our analysis to the most simple dialectical situation, assuming that the author is arguing herself on the background of her own position. ${ }^{3}$ Many other situations are possible, for example, an author may merely report an argument or she may present an argument in the name of somebody else, from the supposed point of view of the audience or on the basis of what she takes to be common ground in a given context. As our discussion of examples (in Sect. 6.1) will show, dealing with such cases indeed is an important topic for further research; but it lies outside the scope of the present paper.

Our guiding question also reflects a particular perspective on arguments. We adopt the point of view of an interpreter. ${ }^{4}$ This contrasts with other approaches that study arguments, for example, as a means to rationally resolve a difference in opinion (e.g. van Eemeren and Grootendorst 2004), to persuade somebody or to provide knowledge (e.g. Lumer 2005). ${ }^{5}$ According to a long tradition in hermeneutics and the philosophy of language, theoretical preconditions of interpretation and considerations of fairness speak in favour of charitable interpretations (Davidson 1984; Scholz 2001). In particular, an interpreter needs to provide precautions against drawing upon inadequate interpretive assumptions when classifying an argument as invalid. An interpreter should try to find an interpretation that makes the argument presented valid. Charitable interpretation also rules out, for example, "completing" an argument by adding a premise that is inconsistent with the explicitly stated premises or by adding the conclusion to the premises and thus turning the argument into a petitio principii. Even a charitable

\footnotetext{
1 Throughout this paper, we refer to authors by female pronouns and to interpreters by male pronouns.

2 This does not commit us to deductivism, the claim that all arguments "really" are deductive, nor to heuristic deductivism (Ennis 1982, pp. 74-76, 86), the strategy of reconstructing arguments as deductively valid as a means for determining "missing" premises. For the sake of simplicity, we will assume that the author and the interpreter do not reason differently in that they acknowledge different logical rules and axioms as valid. Those who think that different logics are in use need to incorporate this variable in their theories of interpretation.

3 This includes arguments that are presented just for the sake of argument. See Sect. 5.

4 In the literature, interpretation is sometimes contrasted with reconstruction as follows: Interpretation is associated with what ordinary language users actually do in dealing with arguments presented by an author, whereas reconstruction is an activity guided by theoretical considerations, standards of evaluation and techniques of argument analysis (cf. van Rees 2001). In this terminology, we deal with reconstruction rather than interpretation.

5 Paglieri and Castelfranchi (2006) propose to use a belief revision framework for analysing how arguments may effect (or fail to effect) belief changes in an addressee in an attempt to persuade him.
} 
interpreter should not reconstruct every enthymeme as valid. We will allow for various degrees of charity. Deciding which degree of charity an interpreter should invest is not a matter of applying a mechanical procedure but requires a judgement about whether it is more plausible to assume that the author made a mistake in presenting her argument as valid or that the interpreter relied on inadequate interpretative assumptions when deeming the argument invalid. Focusing on interpretation means that we need to determine what we are entitled to ascribe to an author on the basis of the arguments that she is presenting in a particular situation. This ascription may be different from what the author in fact believes or what she would accept if it was proposed to her. We are not interested in what the author had better said or believed, but she does not in fact say or believe.

The problem we are going to deal with is the problem of interpreting enthymemes. However, the term "enthymeme" is used in many different ways in historical and contemporary literature (cf. Gerritsen 2001; Walton and Macagno 2009). For the purposes of the present paper, we use enthymeme to refer to an argument that is deductively invalid but a candidate for being interpreted as enthymematically valid. We assume a distinction between three classes of arguments: deductively valid arguments, the conclusions of which must be true if their premises are true; enthymematically valid arguments, which are deductively invalid but become valid if something implicit is taken into account; invalid arguments, which are neither deductively nor enthymematically valid.

The key idea of the view we will develop is that enthymemes need to be evaluated against the background of the author's belief state (this is the "something implicit" just mentioned). It is her belief state that provides the implicit elements responsible for enthymematic validity (Sect. 2). Accordingly, we embed the evaluation of enthymemes into an interpretation strategy that aims at ascribing a belief state to the author (Sect. 3). After briefly presenting some notions of belief revision theory (Sect. 4), we first describe how the ascribed belief state is modified in order to reflect the author's acceptance or denial of the premises and the conclusion (Sect. 5). We then introduce initial tests for distinguishing between enthymematically valid and invalid arguments (Sect. 6.1). If the initial tests are negative, a further step may be undertaken involving additional revisions that offer a way of enforcing enthymematic validityunless the interpreter judges that the ascribed belief state resulting from this move is just too implausible (Sect. 6.2). In the concluding section, we discuss some characteristics of our approach and compare it with the common method of processing enthymemes by adding "missing premises" (Sect. 7).

\section{The basic idea of a belief-revision approach to enthymemes}

The usual approaches to enthymemes aim at specifying a strategy for completing arguments. ${ }^{6}$ They seek to identify "missing premises", that is, they identify one or more

\footnotetext{
6 As Jacquette (1996) argues, adding premises is not always the best strategy for reconstructing an argument as valid. Sometimes it may be more charitable to alter the given argument by strengthening its premises or weakening its conclusion.
} 
additional premises that turn a given invalid argument into a deductively valid one. ${ }^{7}$ For example, it has been argued that "He is an Englishman; he is, therefore, brave" should be completed by "The English are all brave" (van Eemeren and Grootendorst 1982 , p. 104; the example is from Grice 1975, pp. 44-45/1989, p. 25). As an alternative, Paglieri and Woods (2011) recently suggested that enthymemes are in need of completion because without prior completion they are not instances of any scheme of argumentation and hence cannot be assessed at all. In their view, completing an enthymeme may well amount to supplying a premise that makes the resulting argument an instance of an invalid argument scheme, such as affirming the consequent. They suggest to complete "Every Catholic priest is male, so John is a Catholic priest" with "John is male" (Paglieri and Woods 2011, p. 470).

An element common to these approaches is that they ask how to add premises to a given argument in order to render it deductively valid or an instance of some scheme of argumentation. We argue that this picture should be replaced. People always argue on the background of their beliefs, and the vast majority of these beliefs remain implicit at any given point in time. The premises they explicitly express are those beliefs they want to make salient for some specific reason (our theory will say for which reason). These explicit premises are often believed to be true. In other cases, they are put forward only hypothetically or they may even be disbelieved, but can be "added" to the author's beliefs for the sake of argument. In any case, if an enthymeme is put forward, the implicit elements are typically present in the author's belief state. We suggest changing the perspective and submit that the task of interpreting an enthymeme does not consist in adding some implicit elements to the elements explicitly put forward, but in adding the explicit elements to the implicit elements of the author's belief state. In the process of interpreting an argument, an interpreter thus has to ascribe a belief state to the author. ${ }^{8}$ An argument will then be classified as enthymematically valid by the interpreter if its conclusion $c$ is supported by the ascribed belief state $\mathbf{B}$ "together with" its premises $P$. Using $\otimes$ for the (yet to be specified) operation of adding a set of sentences to a belief state, this idea can be written symbolically as follows ${ }^{9}$ :

\section{(1) $\mathbf{B} \otimes P \vdash c$.}

If all the explicit premises of the enthymeme are believed to be true by the authorwhich is a very common case - they do not really have to be added to her belief state. They are already accepted in it. They just get highlighted or emphasized by being

\footnotetext{
7 In practice, interpreters also frequently come across arguments that are incomplete because they lack an explicit conclusion. We neglect such cases, following a common practice in the literature on enthymemes (for an exception see Walton and Macagno 2009).

8 Since working with belief states implies that premises and conclusions are something that one actually can believe, our approach is not applicable to schematic arguments, which contain schematic letters instead of natural language expressions. This is not a serious limitation. Schematic arguments are the province of logicians, and they can be expected to present their schematic arguments non-enthymematically. The literature on enthymemes thus generally does not deal with schematic arguments (but see Paglieri and Woods 2011).

9 We are using the derivability symbol $\vdash$ in a slightly unconventional way, with a belief state rather than a set of sentences to the left of it. (1) means that the set of beliefs supported by, or accepted in, the belief state $\mathbf{B} \otimes P$ entails $c$. We trust that this notational convention will not cause any confusion (cf. also Sect. 4).
} 
explicitly mentioned. We will shortly explain how this limiting case of "adding" can be analysed.

As an example, let us have a look at Turing's "theological objection" against the possibility of thinking machines. It runs as follows. "Thinking is a function of man's immortal soul. God has given an immortal soul to every man and woman, but not to any other animal or to machines. Hence no [other] animal or machine can think" (Turing 1950, p. 443). If we read the first premise as "Having an immortal soul is a necessary condition for thinking", this is not a deductively valid inference. ${ }^{10}$ Besides the possibility mentioned in the second premise, there might be other ways an immortal soul is bestowed on, or acquired by, animals and machines. A traditional analysis would seek to supply an additional premise, such as "Nothing has an immortal soul unless it has been given one by God." Or, stronger, "Nothing has a soul unless it has been given one by God." Or perhaps just the "logical minimum", which is the material conditional with the conjunction of the premises as its antecedent and the conclusion as its consequent. In our analysis, we need not decide between these options. If an author advances the theological objection, her interpreter will charitably seek to ascribe a belief state to her which together with her premises supports the conclusion "No other animal or machine can think."

Ascribing a belief state to an author of an enthymeme means a lot more than just the coming up with some additional premises. As we shall explain in Sect. 4, a belief state encodes a belief set, i.e., a set of sentences that are believed to be true in that belief state, but it also goes beyond the belief set. And the two tasks, evaluating arguments and ascribing belief states, are deeply intertwined. Previous ascriptions of belief states play a role in the evaluation of the current argument, and the result of this evaluation will usually have consequences for the ascription of beliefs to the author. We first introduce a strategy for interpreting arguments.

\section{A strategy for interpreting arguments}

For the interpretation to start somewhere, an initial ascription of a belief state is needed. This belief state will then successively be revised in an iterative process which interprets the author's arguments one after the other.

When an interpreter initially ascribes a belief state to an author, he forms hypotheses about the author's beliefs, subject to later corrections in the course of interpreting the author's arguments. Initial ascriptions include what the interpreter considers to be default beliefs; that is, the beliefs he is ready to ascribe to any author as long as he does not have evidence to the contrary. We take it that the principle of humanity (Grandy 1973) is operative here. The interpreter assumes that the author has the beliefs he himself would have in the author's situation. Equally important for the initial ascription of a belief state are various kinds of contextual evidence, such as information about the author, her language, her epistemic situation, the circumstances of text production,

\footnotetext{
10 A similar point could be made with alternative readings, such as "Having an immortal human soul is a necessary condition for thinking." This interpretation is less charitable. It renders the second "theological" premise superfluous, as we may assume that the author of the argument believes that only men and women can have human souls.
} 
relevant cultural stereotypes etc. This will modify the assumption of default beliefs and result in a tailor-made initial construction of this particular author's belief state. The amount and quality of specific information available may vary from almost nothing, like in the case of text-book arguments, to a wealth of knowledge about an author the interpreter is well-acquainted with.

Only at the very beginning, the interpretation of an argument will proceed against the backdrop of such an externally ascribed belief state (external to the text under consideration). All later interpretations will rely on the ascription of a belief state that has been revised by the interpretation of previous arguments put forward in the text. By the previously ascribed belief state, we refer to the belief state ascribed at the point just before the argument under consideration is presented. It may well happen that the previously ascribed belief state supports the negation of some premises or of the conclusion of the current argument.

Let us now focus on the interpretation of a particular argument. When an author presents an argument, we do not only learn that she draws a certain inference, but also and at the same time she usually communicates whether she accepts or denies its premises and conclusion. We will therefore distinguish two steps in the interpretation of an argument. Firstly, the previously ascribed belief state may have to be adapted in order to account for the acceptance or the denial of the argument's premises and conclusion. Secondly, the argument has to be evaluated regarding its deductive or enthymematic validity. Depending on the outcome, further revisions may be needed to form the resulting belief state which then serves as the newly ascribed belief state on the basis of which the interpretation of the next argument will proceed.

Before discussing these two steps in more detail, let us briefly note some issues we are not going to deal with. Firstly, the techniques of logic and belief revision theory cannot be applied directly to arguments as they are usually presented in ordinary texts. A great deal of preparatory work is needed, but in the present paper, we simply presuppose that all this has already been done. More specifically, we do not go into the questions of how to determine whether the author has actually presented an argument as deductively valid and of how to identify an argument's premises and conclusion. Especially the latter task may require quite extensive reconstructive efforts. Sometimes we have to settle on the exact wording of premises and conclusions, for example, in order to guard against ambiguities. ${ }^{11}$ We further assume that the premises and the conclusion have been adequately formalized in a logical language, and that we possess a logical system which provides an adequate norm for evaluating whether arguments are deductively valid. For showing deductive invalidity, we need to be sure that our formalizations account for all features that are relevant to the deductive validity of the argument (this may indeed be problematic; see the discussion initiated by Oliver (1967) and Massey (1975)). In short, we suppose that the argument at hand has been adequately reconstructed, formalized and evaluated for deductive validity. ${ }^{12}$

\footnotetext{
11 In general, argument reconstruction involves four types of operations: deleting irrelevant elements, adding, reformulating and reordering premises and conclusion. In the present context, we assume that reconstruction does not include "completing" enthymemes by adding premises.

12 For a more extensive discussion of these points see Brun $(2004,2008)$ and Brun and Hirsch Hadorn (2009).
} 
Secondly, the strategy just described simplifies things considerably by suggesting a linear sequence of steps. As we will see, the various steps involve that an interpreter weighs up the merits and demerits of alternative interpretations and takes interpretive decisions. These decisions may later turn out to be infelicitous in the light of the author's subsequent arguments. Similarly, later steps of interpretation may call into question the results of the preparatory tasks. If, for example, the resulting assessment of an argument's validity is implausible, this may speak against the formalization used. Tackling such problems would require a correction mechanism, such as a backtracking or looping structure that permits retroactive revisions of previous decisions. Integrating such a correction mechanism lies outside the scope of this paper.

\section{Some elements of belief revision theory}

This paper is not meant to be an exercise in formal logic, but it will be useful to apply some modest formalization. An argument is characterized by a set $P$ of premises and a conclusion $c$. We generally use capitals for sets of sentences and lower case letters for single sentences. We restrict our attention to ordinary arguments in which premises and conclusion are neither tautological nor contradictory. We suppose that the premise set $P=\left\{p_{1}, \ldots, p_{n}\right\}$ is finite and denote the conjunction $p_{1} \wedge \cdots \wedge p_{n}$ of the premises by $p$. Our language includes the standard connectives of propositional logic and in addition a binary conditional connective $>$. The expression " $a>b$ " should be read as "If $a$ then $b$ ", where the conditional is understood in roughly the same way as it is understood in natural language, so $>$ is certainly stronger than the material conditional $\rightarrow .{ }^{13}$ Let $L$ denote the set of all sentences of the language.

Bold face symbols $\mathbf{B}$ stand for belief states. A belief state $\mathbf{B}$ is assumed to be representable by some formal structure that allows one to retrieve the belief set supported by (or accepted in) $\mathbf{B}$, i.e., the set of sentences believed to be true by someone in belief state $\mathbf{B}$. We assume that the set of beliefs supported by a belief state is logically consistent, and that the logical consequences of beliefs supported by a belief state are also supported by that belief state. While these assumptions would be inadequate if we dealt with occurrent belief or professed belief, they are justified given that we deal with beliefs which may be ascribed to a rational author. But a belief state not only characterizes the set of beliefs it supports, it also has non-propositional, "dispositional" components. In order to guide belief revisions and contractions, it must include some kind of selection structure that helps determining how changes of belief such as revisions and contractions are handled. In order to provide this structure, a belief state $\mathbf{B}$ can be represented by a complete pre-ordering of possible worlds which encodes the relative doxastic plausibility of such worlds. In this modelling, the belief set supported by $\mathbf{B}$ is the set of all sentences true at every world that is maximally plausible according to $\mathbf{B}$. Whenever a belief state appears to the left of the derivability

13 We are aware of the fact that there may not be a unique understanding of conditionals in natural language. We do not want to take a stand here on the question whether the conditional $>$ should be read in the indicative or the subjunctive mood. In any case, > is supposed to be a Ramsey test conditional in the sense explained below. 
symbol $\vdash$ in the following, this will be supposed to indicate that the set of beliefs supported by this state entails the sentence at the right hand side.

We will make some use of the resources of belief revision theory from now on. ${ }^{14}$ Let $\underline{\mathbf{B}}$ be the set of all belief states. There are two types of function: (i) revision functions $*$ taking pairs consisting of a belief state $\mathbf{B}$ from $\underline{\mathbf{B}}$ and a sentence $a$ from $L$ as arguments and yielding revised belief states $\mathbf{B} * a$ as values; and (ii) contraction functions $\div$ of the same format. In the case of revision functions, $a$ is a new piece of information or a hypothetical assumption. $\mathbf{B} * a$ is the result of successfully revising $\mathbf{B}$ by $a$, with the effect that $a$ is actually accepted after the change. In the case of contraction functions, $a$ is a piece of information or an assumption that needs to be given up. $\mathbf{B} \div a$ is the result of successfully withdrawing $a$ from $\mathbf{B}$, with the effect that $a$ is no longer accepted after the change.

We adopt a widely used notation and write $\mathbf{B}+a$ instead of $\mathbf{B} * a$ if $a$ is consistent with $\mathbf{B}$. This operation is called belief addition. As long as $a$ does not contain the conditional connective $>$, we may safely assume that the set of beliefs accepted in $\mathbf{B}+a$ is just the logical closure of the union the sentences accepted in $\mathbf{B}$ and $\{a\}$.

Belief revision is a non-trivial task because the belief set should remain consistent even if the input is inconsistent with the prior beliefs. Belief contraction is non-trivial because the sentence to be withdrawn must not be deductively re-derivable from the remaining beliefs. The two tasks are indeed closely related; many researchers endorse the so-called Levi identity $\mathbf{B} * a=(\mathbf{B} \div \neg a)+a$.

We assume that the conditional connective $>$ can be interpreted by the so-called Ramsey test. A compact formulation of the Ramsey test is this: A belief state $\mathbf{B}$ supports $a>b$ if and only if $b$ is supported in the hypothetically revised belief state $\mathbf{B} * a$. The Ramsey test is notorious for its problems, but there is a number of proposals to solve them in the literature. ${ }^{15}$

\section{Accounting for the author's acceptance or denial of premises and conclusion}

If an author puts forward an argument as deductively valid, she commits herself to a certain relationship between premises and conclusion, namely that the conclusion must be true if the premises are true. However, this does not imply that she actually accepts the premises and the conclusion. We can distinguish three types of arguments with respect to the acceptance status of the premises. In what we call effective arguments, the author actually endorses the premises. In a hypothetical argument, she takes a neutral stance on the premises. ${ }^{16}$ Finally, she may express that she actually rejects the premises and present her reasoning just for the sake of argument. Such arguments we call justfor-the-sake-of-argument arguments, or briefly JFTSOA-arguments. The three types

\footnotetext{
14 For overviews of belief revision theory see Gärdenfors (1988), Gärdenfors and Rott (1995), Rott (2008).

15 The classical references are Ramsey (1931, p. 247, footnote), Stalnaker (1968, p. 102) and Gärdenfors (1979). For information about the problems of the Ramsey test and some ideas how to solve them, see Gärdenfors (1986), Lindström and Rabinowicz (1998), Nute and Cross (2001), Leitgeb (2010) and Rott (2011).

16 This use of the term "hypothetical" must not be confused with the more traditional terminology that labels any argument with an if-then premise a "hypothetical argument".
} 
Table 1 Classification of arguments according to acceptance status of premises and conclusion

\begin{tabular}{|c|c|c|c|}
\hline $\begin{array}{l}\text { author expresses } \\
\text { acceptance of }\end{array}$ & $p$ & neither $p$ nor $\neg p$ & $\neg p$ \\
\hline$c$ & effective arguments & \multirow{3}{*}{$\begin{array}{l}\text { hypothetical } \\
\text { arguments }\end{array}$} & \multirow{3}{*}{$\begin{array}{l}\text { just-for-the-sake- } \\
\text { of-argument } \\
\text { arguments }\end{array}$} \\
\hline neither $c$ nor $\neg c$ & & & \\
\hline$\neg c$ & & & \\
\hline
\end{tabular}

of argument can then be subdivided according to the author's accepting or denying the conclusion (see Table 1).

Accepting $\neg p$ means that the author rejects at least one of the premises in $P$. As the shaded cells indicate, there is only one type of effective argument. If an author presents an argument as deductively valid, she cannot consistently accept its premises and yet take a neutral stance on the conclusion or even reject it. Hypothetical arguments come in two versions. In its pure variety, the author remains uncommitted with respect to the conclusion. But hypothetical arguments can also be presented for a conclusion that is actually endorsed. The author may already have established the conclusion by other means (for instance, by other arguments) and only wants to point out that the same conclusion follows from different premises that she is not able or willing to accept or reject. If a hypothetical argument is presented as deductively valid, the conclusion cannot consistently be rejected. Only in an JFTSOA-argument can the conclusion be rejected. Such arguments are compatible with any stance on the conclusion. After an author has argued for a conclusion, she may present another argument that would establish it if she accepted $p$, which she in fact rejects. But an author may also reason just for the sake of argument from premises she in fact wants to deny to some conclusion she does not accept.

When an argument is presented to us, we usually do not only get information about whether the author considers it to be deductively valid but simultaneously also information about whether she accepts or rejects its premises and conclusion. This information can be given in various degrees of explicitness. Deductive validity is almost never explicitly claimed in a separate sentence. Rather, the conclusion is simply inferred, perhaps marked by an expression like "therefore", "thus" or "so". The situation is different with respect to the author's acceptance or denial of premises and conclusions. If they are phrased as assertions in the indicative mood, we will standardly assume that the author endorses them, unless there is contextual evidence to the contrary. The subjunctive mood, on the other hand, often signals disbelief. Assuming that an author is fully explicit about the acceptance status of her premises and conclusion is an idealization, and in practice problems of text interpretation are to be expected. For our present purposes, we simply presuppose that this interpretative question has been settled and focus on its consequences for the ascription of a belief state to the author.

Whoever is engaged in a project of interpreting an author has to take seriously the question whether she accepts or denies the premises and the conclusion even if he evaluates her argument as invalid. He has to record her stance on premises and 
conclusion in the ascribed belief state. In various situations, the acceptance status the author expresses may conflict with the previously ascribed belief state. If the conflicting ascriptions are initially ascribed beliefs, the current argument just reveals that some of the interpreter's "external" assumptions were wrong and need to be revised. If the conflicting ascriptions are the result of interpreting a previous argument, the current argument reveals either that a mistake was made in an earlier step of interpretation or that the author now takes a stance that is incompatible with what she earlier endorsed. For the sake of simplicity, we assume in both cases that the most recent evidence takes precedence over previous belief ascriptions and that the belief state ascription be revised accordingly. (As mentioned before, more sophisticated strategies would involve a backtracking or looping mechanism for corrections.)

If an argument has been appropriately reconstructed (as explained in Sect. 3), it will be given sentence by sentence. In an effective argument, the author asserts both premises and conclusions, and the interpreter can, if necessary, revise the ascribed belief state $\mathbf{B}$ step-by-step in that order, for example, $\left(\left(\mathbf{B} * p_{1}\right) * p_{2}\right) * c$. In a hypothetical argument, the author may explicitly say something like "I don't know whether $p_{1}$ and $p_{2}$ are true, but if $p_{1}$ and $p_{2}$ are true, $c$ follows." If the previously ascribed belief state supports, say, $p_{1}$ and $p_{2}$, they have to be withdrawn, and the new ascription should be $\left(\left(\mathbf{B} \div p_{1}\right) \div p_{2}\right) .{ }^{17}$ In a JFTSOA-argument, the revision is by the negation of the premises which are marked as disbelieved, and possibly also by the conclusion or its negation. The classification of arguments presented in Table 1 only reflects that the author may accept or reject the conjunction of the premises. This is a simplification since she may of course take a different stance on the various premises, rejecting some of them but endorsing others. ${ }^{18}$

An important point here is that an interpreter can account for the acceptance status of premises and conclusion before he proceeds to test for enthymematic validity itself. Information concerning the acceptance status of the relevant sentences is processed first. Only thereafter, the relation between premises and conclusion is evaluated by the interpreter (a task to which we shall turn in the next section). ${ }^{19}$ As an aside, we may note that this way of proceeding can also naturally deal with the assertion or denial of

\footnotetext{
17 Notice that $\left(\mathbf{B} \div p_{1}\right) \div p_{2}$ is in general different from $\left(\mathbf{B} \div p_{2}\right) \div p_{1}$, and this effect may not be welcome. Simultaneous belief change with respect to two beliefs does not offer a general way out. Simply contracting with the conjunction $p_{1} \wedge p_{2}$ or the disjunction $p_{1} \vee p_{2}$ does not help, since the former will not necessarily eliminate both conjuncts from $\mathbf{B}$ while the latter will not only eliminate the disjuncts but also the disjunctive belief $p_{1} \vee p_{2}$.

18 Arguments involving a "mixed" acceptance status of premises and conclusion are more difficult to deal with because standard belief revision theories can only handle one belief state manipulation at a time. Combining a revision with a contraction may be a problem, because the contraction operation $\mathbf{B} \div p_{i}$ removes not only $p_{i}$ from the belief set supported by $\mathbf{B}$ but also enough of the remaining part of $\mathbf{B}$ to guarantee that $\mathbf{B}$ no longer supports $p_{i}$. Thus when we want to combine a revision with a contraction, the second operation may partly undo the first. For example, contracting with $p_{2}$ will possibly eliminate $p_{1}$ if we perform $\left(\mathbf{B} * p_{1}\right) \div p_{2}$.

19 This can of course be disputed, as the order of processing the statement of premises and conclusion and the transition from the former to the latter does not appear to be fixed. We do not have strong reasons why an interpreter should account for the acceptance/rejection of premises and conclusion before evaluating the enthymematic validity of the argument. But our suggestion has at least the advantage of being the simplest solution.
} 
an individual sentence not embedded in an argument pattern. For its interpretation, it can be treated as if it were the conclusion of a deductively valid argument with zero premises.

\section{Evaluating enthymemes}

As explained before, interpretative charity motivates classifying some arguments that are not deductively valid as enthymematically valid. We now have to address the questions of what the conditions are for an argument to be enthymematically valid, and of what consequences such an evaluation may have for the ascription of a belief state to the author.

\subsection{The first step: initial tests for enthymematic validity}

According to the basic idea outlined in Sect. 2, testing for enthymematic validity does not amount to guessing which missing premises might be added to $P$ in order to make the inference from $P$ to $c$ deductively valid. Instead, we consider the whole of the author's ascribed belief state $\mathbf{B}$ as the context against which the argument has to be evaluated. From now on, we take $\mathbf{B}$ to be the belief state which has been adapted to account for the acceptance or rejection of the premises and the conclusion. Our first attempt at fleshing out the general scheme (1) with more specific content uses the operation of addition in the sense of belief revision theory. The argument from $P$ to $c$ is enthymematically valid (relative to $\mathbf{B}$ ) if and only if

(2) $\mathbf{B}+p \vdash c$.

This basic idea needs to be adapted in two classes of cases:

(Inconsistency) $\mathbf{B}$ and $p$ are inconsistent with one another; that is, $\mathbf{B}$ supports $\neg p$. (Irrelevance) $\quad \mathbf{B}$ alone supports $c$ without the help of $p$.

(Inconsistency) characterizes the situation of JFTSOA-arguments. The author makes it clear in the text that she rejects $p$, i.e. she rejects at least one element of $P$, and puts forward the inference from $P$ to $c$ just for the sake of argument. In this case, we can replace (2) by a condition that uses revision:

(3) $\mathbf{B} * p \vdash c$.

Condition (3) reduces to (2) if $\mathbf{B}$ and $p$ are consistent with one another.

(Irrelevance) covers the complications that arise if $\mathbf{B}$ itself supports $c$. This can happen, for example, if the conclusion of an argument is already believed because the author has presented another argument for the same conclusion before (as, for instance, Thomas Aquinas does in his Five Ways). The premises $P$ may be, but need not be supported by $\mathbf{B}$ as well. Either way, we improve on (2) by taking a condition that uses contraction: 
(4) $(\mathbf{B} \div c)+p \vdash c$.

The motivation for (4) is that if $\mathbf{B}$ already supports $c$, then (2) is trivially met for any $p$ that can be added consistently to $\mathbf{B}$. To make sure that the premises $P$ are relevant for the validity of the enthymeme, we suggest to test whether $c$ is supported by $p$ added to the contracted state $\mathbf{B} \div c$ that does not support $c$ any more. As we know from Sect. 4 , $(\mathbf{B} \div c)$ removes not only $c$ itself from $\mathbf{B}$ but enough of the remaining elements of $\mathbf{B}$ to guarantee that the resulting state no longer supports $c$. Condition (4) reduces to (2) if $c$ is not supported by $\mathbf{B}$.

Arguments that fall under both (Inconsistency) and (Irrelevance) are particularly interesting. Approached from (Inconsistency), one may expect the condition $\mathbf{B} * p \vdash c$ to apply, but approached from (Irrelevance), one expects $(\mathbf{B} \div c)+p \vdash c$. It is evident that (3) does not solve the irrelevance problem, and since the contracted belief state $\mathbf{B} \div c$ may still support $\neg p$, (4) does not solve the inconsistency problem. So we suggest to use the following condition that takes care of both (Inconsistency) and (Irrelevance):

(5) $(\mathbf{B} \div c) * p \vdash c$.

An example helps to make clear why $(\mathbf{B} \div c) * p \vdash c$ may be needed in cases in which both (Inconsistency) and (Irrelevance) apply. Imagine an author who gives a JFTSOA-argument with premise $p$ for $c$, having already argued that $c$ follows from other premises $r$ and $r \rightarrow c$. That is, she argues that $c$ would follow even if she accepted $p$ (which she in fact rejects). Furthermore, assume that the interpreter ascribes to the author a belief state $\mathbf{B}$ that supports $p \rightarrow c, r, r \rightarrow c, c$ and $\neg p$. Since $\mathbf{B}$ supports $c$, (Irrelevance) applies, and since it supports $\neg p$, (Inconsistency) applies as well. If the interpreter uses condition (3), the consistent revision of $\mathbf{B}$ by $p$ will remove $\neg p$, but it will presumably still support $r$ and $r \rightarrow c$ and thus continue to support $c$. This is undesirable since it means that $p$ is irrelevant for the derivation of $c$; many arbitrary premises consistent with $c$ would pass this test (3). If the interpreter uses condition (4), he will run into the problem that $\mathbf{B} \div c$ will presumably still support $\neg p$ and become inconsistent once $p$ is added. Both problems vanish if condition (5) is used, because the revision of $\mathbf{B} \div c$ by $p$ will not lead into inconsistency and effectively require that $p$ be relevant for $c$.

Table 2 provides an overview of the variations of the test considered so far. We call it initial test, because we will suggest that the interpretation process should provide further steps if this test fails.

Table 2 Initial tests for enthymematic validity

\begin{tabular}{|c|c|c|c|}
\hline B supports & $p$ & neither $p$ nor $\neg p$ & $\neg p$ \\
\hline$c$ & $(\mathbf{B} \div c)+p \vdash c$ & $(\mathbf{B} \div c)+p \vdash c$ & $(\mathbf{B} \div c) * p \vdash c$ \\
\hline neither $c$ nor $\neg c$ & & $\mathbf{B}+p \vdash c$ & $\mathbf{B} * p \vdash c$ \\
\hline$\neg c$ & & & $\mathbf{B} * p \vdash c$ \\
\hline
\end{tabular}


Condition (5) is the universally applicable test for enthymematic validity. It simplifies to conditions (2), (3) or (4), respectively, whenever the problems (Irrelevance) and (Inconsistency) are absent. Our official definition at this point of the analysis is this: An argument from a set of premises $P$ to a conclusion $c$ is enthymematically valid if and only if the previously ascribed belief state $\mathbf{B}$ satisfies the test condition $(\mathbf{B} \div c) * p \vdash c$. If the test succeeds, the interpretation of this argument is finished.

Two important points need to be noted. Firstly, when executing the operations of $+p, * p$ and $\div c$ specified in conditions (2)-(5) the interpreter is just playing "let's pretend". These operations are used to test for enthymematic validity. The resulting belief states are purely hypothetical, because the author does not in fact come to accept $p$ or to reject $c$ in the relevant situations. The revised belief states listed in Table 2 are not ascribed to the author by the interpreter. If the initial test is successful, the argument is accepted as enthymematically valid relative to $\mathbf{B}$, and belief state $\mathbf{B}$ continues to be ascribed to the author.

Secondly, the tests we have described are just that-tests. They do not suggest to identify a "completed" argument. When checking whether the belief set supported by the belief state $(\mathbf{B} \div c)+p$ entails $c$, we are not suggesting to tamper with the argument consisting of the premises $P$ and the conclusion $c$; the argument remains as it was originally put forward. If an argument passes the initial test, the author is ascribed the belief state $\mathbf{B}$. Of course, $\mathbf{B}$ may support one or many sentences $s$ which could be used to construct a new, deductively valid argument with the premises $P \cup\{s\}$ and the conclusion $c$. However, this does not place us in the same boat with the find-themissing-premise strategy. We are neither committed to answering the question which of the sentences supported by B are "really" the ones that should complete argument. We deny that the author somehow managed to advance a specific argument that differs from the one she in fact presented.

The tools developed so far suffice to deal with a wide range of textbook examples of enthymemes. Specifically, our tests will underwrite the enthymematic validity of so called "materially valid" (Sellars 1953) or "analytic" inferences such as "Henry is a frog; therefore Henry is not a man" (Davidson 1980, p. 143). Typically, the relevant generalization ("No frog is a man") will be taken to be accepted in the initially ascribed belief state. Whether this is actually the case depends on the interpreter's own knowledge and the assumptions he used for building up the initially ascribed belief state. It may not include what is needed to account for the enthymematic validity of "Socrates was a hoplite; therefore he was a soldier" (cf. Paglieri and Woods 2011, pp. 471-472).

Let us now consider two more tricky examples before we address the case of unsuccessful initial tests.

Example 1 Consider a situation in which two conditions are individually necessary and jointly sufficient for a certain proposition to obtain. ${ }^{20}$ For instance, let us assume that it is common knowledge (in a given situation) that a candidate will get tenured if and only if her teaching performance and her research output are judged excellent.

\footnotetext{
20 We thank Eduardo Fermé for coming up with the following variant of this example. Someone says: " $y$ is odd. Therefore the sum of $x$ and $y$ is odd, too". Every charitable interpreter will construe this as an enthymeme, assuming that the author somehow believes or knows that $x$ is even.
} 
An author (speaker) gives the following enthymematic argument: "Sarah's teaching is excellent. Therefore she will get tenure." A charitable interpreter will tend to construe this inference as enthymematically valid, and the relevant unexpressed belief is obviously "Sarah's research is excellent." Does our model predict this result? Since the speaker makes two assertions in the indicative mood, she believes that Sarah is an excellent teacher and that she will get tenure. Knowing that excellence in research is also necessary for tenure, the interpreter construes the author as believing that Sarah's research is excellent as well. All this is in the ascribed belief state B. Condition (5) tells us that we first have to contract $\mathbf{B}$ by "Sarah will get tenure." Since this should not result in a retraction of the common knowledge about the requirements for tenure, the contraction will need to eliminate (at least) one of the sentences "Sarah's teaching is excellent" and "Sarah's research is excellent." If the former is kept, then adding it as a premise does not change anything, and condition (5) is violated. If, however, the latter is kept, then adding "Sarah's teaching is excellent" will be sufficient to get back that Sarah will get tenure, and thus (5) is satisfied. So our proposal is adequate in this case if and only if the premise $p$ ("Sarah's teaching is excellent") is lost after a contraction of $\mathbf{B}$ with respect to the conclusion $c$ ("Sarah will get tenure").

It is a consequence of our approach, then, that the premises which are explicitly mentioned in an enthymematically valid argument are exactly those premises of a corresponding deductively valid argument which are least entrenched in the belief state. While this is certainly not implausible, it is a very stringent requirement. In the practice of argumentation, it is not only the belief state ascribed to the author that serves as a point of reference. As an alternative, we can imagine that the author argues on the basis of the belief state that she ascribes to the addressee. Or else she may base her arguments on what she takes as common belief, common knowledge or common ground (Clark 1996, pp. 92-121; Stalnaker 2002) among the participants of the conversation. The corresponding extension or revision of our model will have to wait for further research. In the present paper, we focus on the simplest case, in which everything revolves around the belief state ascribed to the author.

Example 2 Consider the following situation. ${ }^{21}$ After suitable formalization, the argument in question has the single premise

(Law) If there is a lightning, there will be thunder soon after. $\forall t\left(\right.$ lightning $(t) \rightarrow \exists t^{\prime}\left(\right.$ soon-after $\left(t^{\prime}, t\right) \wedge$ thunder $\left.\left.\left(t^{\prime}\right)\right)\right)$

and the conclusion

(Fact) There will be thunder soon. thunder $\left(i^{\prime}\right)$

where $i^{\prime}$ is some point of time shortly after the time at which the speaker utters the argument. It seems obvious that this argument needs a premise like "There was a lightning just now.", or lightning $(i)$, with $i$ not long before $i^{\prime}$, in order to be converted into a deductively valid argument. Suppose that the previously ascribed belief state $\mathbf{B}$ in fact supports lightning $(i)$, and also (Law) and (Fact). The example

21 We thank Ralf Busse for coming up with this example. It originates with Sellars (1953, p. 323). 
seems to show that (5) is the wrong test. The objection runs as follows. According to (5), the argument is enthymematically valid if and only if $\left(\mathbf{B} \div \operatorname{thunder}\left(i^{\prime}\right)\right) *$ (Law) $\vdash$ thunder $\left(i^{\prime}\right)$. Suppose the interpreter withdraws thunder $\left(i^{\prime}\right)$ from $\mathbf{B}$. It is plausible to assume, so the objection continues, that laws are better entrenched than facts, so (Law) is better entrenched than lightning $(i)$. But then $\mathbf{B} \div \operatorname{thunder}\left(i^{\prime}\right)$ is bound to lose lightning( $i$ ) and keep (Law). Subsequent revision by (Law) does not change anything, and thunder $\left(i^{\prime}\right)$ cannot be derived. So the test fails, although intuitively, the enthymeme looks perfectly acceptable. After all, B supports lightning $(i)$ and hence accepting (Law) suffices for deriving thunder $\left(i^{\prime}\right)$.

The objection draws on the assumption that (Law) is better entrenched in $\mathbf{B}$ than lightning $(i)$. This idea is consistent with the fact that it is fairly common to present an enthymeme like

There was a lightning just now. Therefore, there will be thunder soon.

But this is not the counterexample we are discussing now. Our example describes a special context, in which the author herself seems to assume that it is better to mention (Law) rather than lightning(i). (Law) seems to be more dubious than lightning(i) dubious for the author herself or dubious for the intended audience as perceived by the author or dubious with respect to the common ground. ${ }^{22}$ Such a situation may arise, for instance, if both speaker and audience have just witnessed an impressive lightning, and hence the potential absence of any thunder cannot be due to the absence of a lightning. In short, given that the author explicitly presents (Law) as a premise but does not mention lightning( $i$ ), it is implausible to assume that (Law) is better entrenched than lightning $(i)$ in her belief state $\mathbf{B}$ (or in what she thinks is the hearer's belief state, or in what she thinks is the common ground). So we deny what the objection simply stipulates, namely, that (Law) is better entrenched in $\mathbf{B}$ than lightning $(i)$. This reply draws attention to a complication that we have already come across in the previous example. The author's own point of view is not necessarily the ultimate reference point for the interpretation of her arguments, because she may not want to rely on it for the purpose of her argument. Ultimately, an interpretation of an enthymematic argument may not only need to account for the author's own belief state but also for her anticipation of the belief states of the readers (or hearers) or for the common ground.

Finally, we need to mention some techniques of arguing which raise further issues. Argument structures like reasoning by cases or reductio ad absurdum make use of assumptions which are "active" for one or more argumentative steps before they get discharged. Although assumptions clearly have a just-for-the-sake-of-argument flavour, they differ in an important way from the premises used in JFTSOA-arguments as characterized above. In advancing a sentence $q$ as an assumption, the author does not give any information about $q$ 's acceptance status. Assumptions thus call for modifications of the ascribed belief state which are purely hypothetical, but may remain in place for several argumentative steps. Reductio arguments in addition raise special problems. ${ }^{23}$ Typically, the author gives conversational signals ("suppose for reductio") that she

22 If the author presents the argument to a child, she may think that (Law) is unknown to him.

23 We thank an anonymous reviewer of this journal for raising this point. 
is about to present a reductio argument, and such signals tell the interpreter that he should, in this special case, not restore consistency when he performs the hypothetical revision of the ascribed belief state by the assumption. He is rather supposed to expand the ascribed belief set by the assumption, and then to witness how it leads to an inconsistency-just in order to discharge the assumption and replace it by its negation. Thus, even if (Inconsistency) is fulfilled, the interpreter must stick to the basic idea (2) and must not replace it by (3); that is, he must proceed as if the premises were consistent with the author's belief state. Clearly, our account needs to be extended in order to deal with assumptions, but such extensions lie outside the scope of the present paper.

\subsection{The second step: potential revisions of the ascribed belief state}

If the initial test for enthymematic validity is successful, the interpretation of the argument at hand is finished, and we may move on to the next argument in the way described in Sect. 3. But how should we proceed if the initial test fails? That is, if an argument from $P$ to $c$ does not satisfy condition (5)? Does this establish that the argument must be considered as invalid? We do not think so. Interpretation ought to be more charitable. In many cases, it is more likely that the interpreter's ascription of the belief state $\mathbf{B}$ to the author is inaccurate than that the author's argument is invalid. The interpreter has reached a decision point. We suggest that a good interpreter should try to find a charitable revision $\mathbf{B}^{\prime}$ of $\mathbf{B}$ on the basis of which the argument at hand is enthymematically valid. This means that we are now advancing to a second level of charity. In the preceding sections, the idea of charity called for not simply rejecting every enthymeme as invalid. In this section, we discuss how an interpreter can be even more charitable and revise the result of the procedure applied on the first level if it does not attest to the enthymeme's validity. As it turns out, the interpreter can do this by revising the belief state ascribed to the author.

The case in which $c$ is not already supported in $\mathbf{B}$ is relatively easy to deal with. If the initial test $\mathbf{B} * p \vdash c$ is not satisfied, we can construct a minimal change $\mathbf{B}^{\prime}$ such that $\mathbf{B}^{\prime} * p \vdash c$ is satisfied. The recipe is to minimally change $\mathbf{B}$ in such a way as to satisfy the conditional $p>c$ :

$$
\mathbf{B}^{\prime}:=\mathbf{B} *(p>c) .
$$

The revision function $*$ guarantees that $\mathbf{B}^{\prime}$ supports $p>c$, and by the Ramsey-test analysis of conditionals (cf. Sect. 4), this means that $\mathbf{B}^{\prime} * p \vdash c .{ }^{24}$ Hence, $\mathbf{B}^{\prime}$ is a belief

\footnotetext{
${ }^{24}$ Revising by the material conditional $p \rightarrow c$ would not suffice. For instance, a revision by $p \rightarrow c$ has no effect if $\mathbf{B}$ already supports $\neg p$, so this method cannot be used for JFTSOA-arguments. However, revising a belief state by the (stronger) conditional $p>c$ is not an easy task. In the theory of belief revision, research on this problem has begun in the 1990s (Boutilier and Goldszmidt 1995; Nayak et al. 1996; Kern-Isberner 1999). The revision by the conditional $p>q$ is guaranteed to effect a restructuring of a belief state within the $p$-worlds, and thus has far-reaching consequences in the "dispositional" part of the belief state. The only thing we need here is the idea that after a revision by $p>c$, the possible worlds satisfying $p \wedge \neg c$ are set back in terms of doxastic plausibility. But the details of all this are dependent on the specific method used for revisions by conditionals.
} 
state that fulfils the role required by condition (3). Of course, the crucial question is whether we succeed in showing that the transition from $\mathbf{B}$ to $\mathbf{B}^{\prime}$ is well-motivated. Does this proposal not just mean that the interpreter is forcing what he wishes to have, without respecting the evidence that had helped him forming the ascription of the belief state $\mathbf{B}$ in the first place? Is he not over-charitable here, to the point of being irrational? There is no ready-made answer to these questions. As mentioned above, the interpreter has to compare the respective merits of the assumption that he has misconstrued the author's belief state and the assumption that the author committed a fallacy (in the sense of presenting an invalid argument as valid). This can be reflected by our model in the following way. On the one hand, if $\mathbf{B}^{\prime}$ is, intuitively speaking, very far from $\mathbf{B}$, then it is comparatively unlikely that $\mathbf{B}^{\prime}$ represents the real beliefs of the author, and it is more likely that she committed a fallacy. This case applies, for instance, if $\mathbf{B}^{\prime}$ ascribes "crazy" beliefs to the author. Should this be the case, it is preferable to deem the argument at hand to be invalid, and to carry on with the ascription of $\mathbf{B}$. The argument about the Catholic priest John is a case in point (cf. Sect. 2). Revising the ascribed belief state with "If every Catholic priest is male, then John is a Catholic priest" would mean that the interpreter takes the author to be incapable (or, at any rate, hardly capable) of imagining a world at which all Catholic priests are male but John is not a Catholic priest. It would be rather uncharitable to ascribe such a belief state to the author, less charitable than interpreting the argument "Every Catholic priest is male, so John is a Catholic priest" as invalid. If, on the other hand, $\mathbf{B}^{\prime}$ is a comparatively mild and reasonable correction of $\mathbf{B}$, then it is likely that $\mathbf{B}^{\prime}$ is a belief state that is sufficiently faithful both to the interpreter's earlier considerations concerning the author's belief state and to the presumption that the argument from $P$ to $c$ is enthymematically valid. In that case, it is preferable that he accepts the argument as enthymematically valid and ascribes the belief state $\mathbf{B}^{\prime}$ to its author. In this vein, an interpreter may revise the ascribed belief state with "If Socrates was a hoplite, then he was a soldier" rather than deeming "Socrates was a hoplite; therefore he was a soldier" invalid. In our approach, an intuitive judgement of similarity between belief states is involved in deciding whether $\mathbf{B}$ should be revised or the argument should be deemed invalid. In this respect, there is an important contrast to the initial tests described in the preceding section. Whereas these tests consist in applying a formal criterion, deciding whether $\mathbf{B}^{\prime}$ is similar enough to $\mathbf{B}$ requires judgement. The literature offers a variety of notions appropriate to measure similarities between belief states, but we do not think it is necessary for our purposes to take a stand on which of these notions might perform best in the present context. ${ }^{25}$ The "enough" in the phrase "similar enough" provides more leeway for the interpreter's judgement than the amount of rigidity which could be gained by a precise formal explication of what counts as "similar".

So far we have addressed the case in which the conclusion of the argument is not accepted prior to the argument. Much more difficult is the case of a belief state $\mathbf{B}$ that already supports $c$. As we argued above, we have to consider the contracted belief state $\mathbf{B} \div c$ in order to solve the problem of irrelevant premises. But what should an

25 See Katsuno and Mendelzon (1991), Hansson (1992), Peppas et al. (2000), Lehmann et al. (2001), Konieczny and Pino-Pérez (2002) and Arieli et al. (2007). Thanks to one of our reviewers for pressing us to be explicit in acknowledging the fundamental role that the similarity of belief states plays in our proposal. 
interpreter do if it turns out that condition (5) does not hold? Again, we think that it would be premature to conclude that the inference from $P$ to $c$ is just invalid. An interpreter can be more charitable and consider the state

$$
\mathbf{B}^{\prime \prime}:=(\mathbf{B} \div c) *(p>c)
$$

The revision function * guarantees that $\mathbf{B}^{\prime \prime}$ supports $p>c$, and by the Ramsey-test analysis of conditionals, this means that $\mathbf{B}^{\prime \prime} * p \vdash c$. Hence, $\mathbf{B}^{\prime \prime}$ is a belief state that fulfils the role required by condition (3). Let us again assume that we are able to pass an intuitive judgement concerning the similarity of $\mathbf{B}^{\prime \prime}$ to $\mathbf{B} \div c$ which guides the decision whether the initial failure to validate the argument was due to a wrong belief ascription on the side of the interpreter or to invalid reasoning on the side of the author. If we decide for the latter, then we will reject the enthymeme as invalid and we will again stick to ascribing $\mathbf{B}$ to its author. If, however, we decide for the former, then we deem it appropriate to say that the argument is enthymematically valid. It seems that the situation is similar to the previous one in which $c$ is not believed in B. But unfortunately, it is a lot more complicated, since the withdrawal of $c$ from the ascribed belief state $\mathbf{B}$ was purely hypothetical, and the result $\mathbf{B} \div c$ must not be used for the ascription of actual beliefs to the author. So what is the point of having found a variant $\mathbf{B}^{\prime \prime}$ of $\mathbf{B} \div c$ which is suitable for validating the enthymematic inference from $p$ to $c$ ? The interpreter has constructed a variant of the hypothetically contracted belief state $\mathbf{B} \div c$, but he does not have a variant of the factually ascribed belief state $\mathbf{B}$ itself. Now in belief revision theory, it is often held that the original belief state $\mathbf{B}$ can be recovered from the contracted state $\mathbf{B} \div c$ by adding $c$ again, provided that $\mathbf{B}$ supports $c$ in the first place. It follows in fact from the most widely accepted axioms that $(\mathbf{B} \div c)+c=\mathbf{B}$ if $c$ is supported by $\mathbf{B} .{ }^{26}$ So if $\mathbf{B}^{\prime \prime}=(\mathbf{B} \div c) *(p>c)$ is a good approximation of $\mathbf{B} \div c$ that supports the argument from $P$ to $c$, then it seems justified to think that $\mathbf{B}^{\prime \prime}+c$ is a good approximation of $(\mathbf{B} \div c)+c=\mathbf{B}$. The motivating idea is that adding $c$ to two belief states that are close to each other should lead to results that remain close to each other. If we accept this line of thought, then $\mathbf{B}^{\prime \prime \prime}=\mathbf{B}^{\prime \prime}+c=((\mathbf{B} \div c) *(p>c))+c$ is a belief state that may be ascribed to the author by a charitable interpreter of the argument from $P$ to $c$, provided that $c$ is present in the previously ascribed belief state B. ${ }^{27}$ So we have managed to deal with the important case in which the conclusion $c$ of the argument under consideration is believed in $\mathbf{B}$.

An example will make clearer what the transition from $\mathbf{B}$ to $\mathbf{B}^{\prime \prime \prime}$ may involve. We start with a plausibility pre-ordering of possible worlds that characterizes a belief state $\mathbf{B}$ supporting $c$. Then we construct, step-by-step, the belief states $\mathbf{B} \div c, \mathbf{B}^{\prime \prime}=$ $(\mathbf{B} \div c) *(p>c)$ and $\mathbf{B}^{\prime \prime \prime}=((\mathbf{B} \div c) *(p>c))+c$. According to what we have

\footnotetext{
26 Here we refer to the axioms introduced by Alchourrón, Gärdenfors and Makinson (see the references given in footnote 14 above). However, the axiom most relevant in the present context, "recovery", is very controversial. Note that in ordinary belief revision theory, belief states are identified with belief sets.

27 A much easier solution would be to take simply $\mathbf{B} *(p>c)$ rather than $(\mathbf{B} \div c) *(p>c)+c$ as the charitably ascribed belief state. But it is not clear, for instance, whether $\mathbf{B}^{-}:=(\mathbf{B} *(p>c)) \div c$ would validate $\mathbf{B}^{-}+p \vdash c$. It would be interesting to study the connection between $\mathbf{B} *(p>c)$ and $(\mathbf{B} \div c) *(p>c)+c$ more closely, but this is a complex task and cannot be done here.
} 


\begin{tabular}{|c|c|c|c|}
\hline \multicolumn{4}{|c|}{ B } \\
\hline \multicolumn{2}{|c|}{$c$} & \multicolumn{2}{|c|}{$\neg c$} \\
\hline 4 & 4 & 4 & 4 \\
\hline 3 & 3 & 3 & 3 \\
\hline 2 & 2 & 2 & 2 \\
\hline 1 & 1 & & \\
\hline$p$ & $\neg p$ & $p$ & $\neg p$ \\
\hline
\end{tabular}
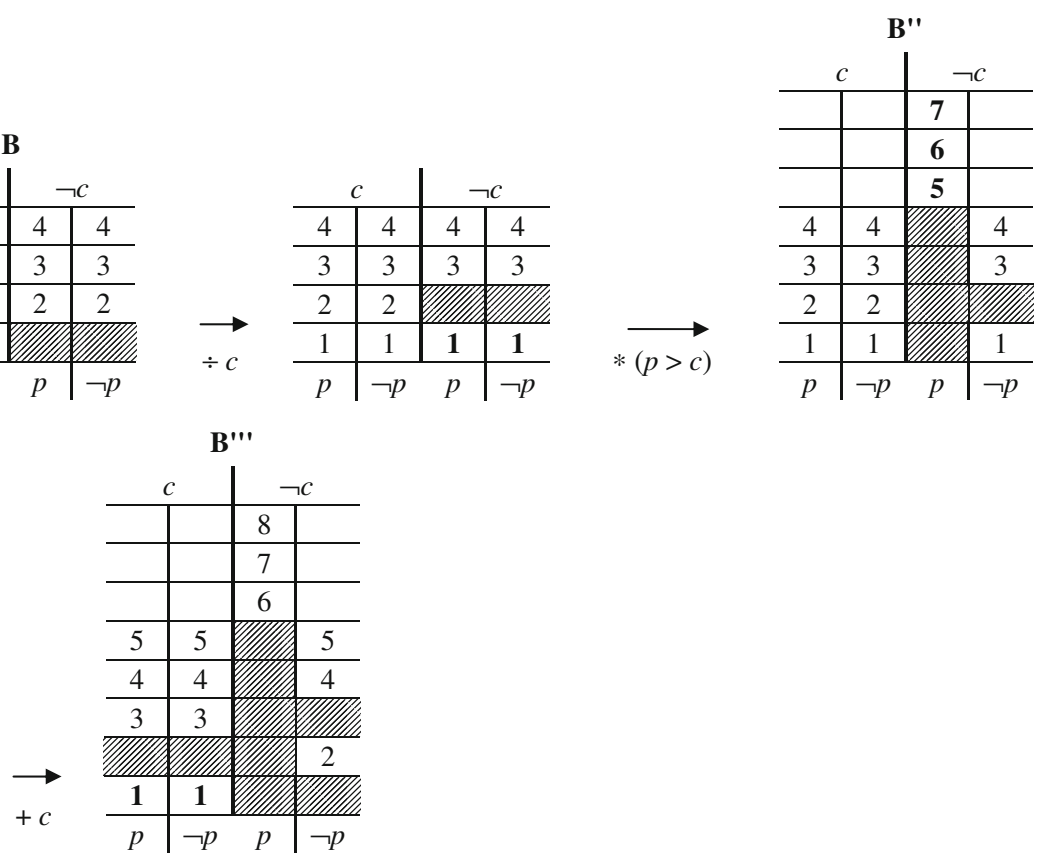

Fig. 1 Example of a sequence of belief states $\mathbf{B}, \mathbf{B} \div c, \mathbf{B}^{\prime \prime}=(\mathbf{B} \div c) *(p>c)$ and $\mathbf{B}^{\prime \prime \prime}=((\mathbf{B} \div c) *$ $(p>c))+c$

just said, the latter state, if reasonably close to $\mathbf{B}$, is the state ascribed at the end of the interpretation process. Figure 1 illustrates how this might happen.

Explanation: The cells represent classes of possible worlds (models, interpretations) that satisfy the sentences indicated at the top and the bottom of the tables. The numbers in the cells indicate positions in the pre-ordering of the possible worlds in terms of plausibility. Smaller numbers indicate higher plausibility, but these numbers should not be taken as carrying information about the distances in terms of plausibility. ${ }^{28}$ Worlds labelled " 1 " are most plausible worlds. Cells with labels in bold faces have been shifted by the last operation. Operations that change the belief state are indicated by arrows. In this example, we use particular contraction and revision functions. The operations $\div c$ and $+c$ applied in the figure are "conservative" contraction and revision operations respectively (see Boutilier 1996 for + , Rott 2009 for $\div$ ). The revision by a conditional, $*(p>c)$, uses an improved version of Nayak et al.'s (1996) lexicographic (also known as "moderate") method for the revision by the corresponding material conditional. The choice of these methods may of course be called into question, and they represent just a few of many possible options. At this stage of our research, the important thing is that there are such methods which can be applied in concrete examples and can be

28 We could express distances if we used Spohn's $(1988,2012)$ model based on ranking functions. This would give us much more powerful tools for constructing contractions, but this model does not only take propositions as inputs, but also needs numbers qualifying the inputs, and such numbers are not provided in spoken or written arguments. 
further scrutinized to assess the merits and demerits of our analyses. Figure 1 also shows why $\mathbf{B}$ does not, but $\left.\mathbf{B}^{\prime \prime \prime}=(\mathbf{B} \div c) *(p>c)\right)+c$ does explain the relevance of $p$ to $c$. In $\mathbf{B}^{\prime \prime \prime}$, worlds satisfying $p$ but falsifying $c$ are considered very implausible.

In Sect. 1, we said that enthymemes are deductively invalid but become enthymematically valid if something implicit is taken into account. We are now in a position to refine our official definition of Sect. 6.1. We can now distinguish two "degrees of enthymematic validity", corresponding to the two degrees of interpretive charity we have been analyzing. Assuming that $\mathbf{B}$ refers to a belief state that has already been adapted to reflect the acceptance status of the premises and the conclusion as expressed by the author in her argument, the following definitions can be used. An argument with a finite set of premises $P$ and a conclusion $c$ is enthymematically valid in the narrower sense, relative to a (previously ascribed) belief state $\mathbf{B}$, if and only if the test condition $(\mathbf{B} \div c) * p \vdash c$ is satisfied. If this test succeeds, the interpretation of this argument is finished at the first level of charity. If the test fails, the interpreter checks whether it is more plausible to revise his belief state ascription to the author than to assume that the author has advanced an invalid argument as valid. If the answer is "yes", then the argument is enthymematically valid and the ascribed belief state is adjusted accordingly. If the answer is "no", then the second level of charity has failed, too, and thus the argument is invalid. The following definition makes this idea more precise and summarizes our proposal. It captures, we suggest, a reasonable notion of enthymematic validity. An inference from $P$ to $c$ is enthymematically valid in the wider (more charitable) sense, relative to a (previously ascribed) belief state $\mathbf{B}$, if and only if either (i) it is enthymematically valid in the narrower sense relative to $\mathbf{B}$, or (ii) $\mathbf{B}$ does not support $c$ and $\mathbf{B}^{\prime}:=\mathbf{B} *(p>c)$ is similar enough to $\mathbf{B}$, or (iii) $\mathbf{B}$ supports $c$ and $\mathbf{B}^{\prime \prime}:=(\mathbf{B} \div c) *(p>c)$ is similar enough to the contraction $\mathbf{B} \div c$. In case (ii), the belief state ascribed to the author will be $\mathbf{B}^{\prime}$ rather than $\mathbf{B}$; in case (iii), it will be $\mathbf{B}^{\prime \prime}+c$ rather than $\mathbf{B}$. Assessing whether a given belief state is a similar enough to another one requires both a workable concept of similarity between belief states and good judgement in the decision how similar is similar enough.

In both variants, the notion of enthymematic validity is relativized to the author's belief state (in contrast to the notion of deductive validity, which is not relativized in this way). Hence the same argument may well be enthymematically valid if presented by one author, but invalid if presented by another. In this sense, the notion of enthymematic validity is a subjective notion. Is enthymematic validity also relativized to the interpreter's perspective? We believe that there are two possible answers here. First, throughout this paper we have emphasized the interpreter's task of ascribing a belief state to an author. In so far as there may be different legitimate ascriptions, there may be different verdicts on the enthymematic validity of an argument, without one verdict being more correct than the other. However, this does not mean that anything goes. Interpreters are bound by the described methodology.

But secondly, one may still think that speaking of "validity" implies more objectivity and cannot be squared with so much variation. In order to reflect this intuition, one could hold that what matters is not the belief state which an interpreter ascribes to the author, but the belief state the author is actually in. According to this conception, an interpreter may easily be mistaken about the author's belief state and thus also in his judgement about an argument's enthymematic validity. But, so this line of reasoning 
goes, since there is no additional subjectivity imported from the side of the interpreter, only one interpretation can be right. However, belief states are complex entities and not open to immediate inspection. This explains why two skilful interpreters may disagree, with perfectly good justification, about the enthymematic validity of a given author's enthymeme, in both the narrower and the wider sense.

\section{Conclusion}

We have approached the problem of enthymemes from the perspective of an interpreter and presented an alternative to the traditional find-the-missing-premise view. It is not necessary, in order to show that an argument is enthymematically valid, to identify specific additional premises that turn it into a complete, deductively valid argument. In our approach, enthymemes need not be altered or supplemented in order to become valid. There is no fact of the matter which premises are "missing", and so no missing premises can or should be read off from the form of an argument. Enthymemes are rather evaluated "holistically"; that is, in the context of the author's whole belief state, as ascribed by the interpreter. Understanding enthymematic arguments is rather a natural part of the interpretation of any author's utterances. Roughly speaking, we call an argument enthymematically valid if and only if its conclusion is supported by the belief state that is ascribed to its author by an interpreter who aims at ascribing a rational and suitably adapted belief state that takes account of the information explicitly given by the author, in particular, of the premises of the argument at hand.

The suggested analysis involves an important shift in focus. What is difficult about interpreting enthymemes is not the question of what argument exactly an interpreter should substitute for the enthymematic argument put forward by the author. This argument should be taken at face value. The central tasks of the interpreter's project are rather to ascribe a belief state to the author and to adapt that ascribed belief state in the process of interpreting a text. The project of keeping track of the author's doxastic state while going through a text goes far beyond the confines of interpreting an individual sentence or argument. The interpreter needs to keep apart two different kinds of changes to the ascribed belief state. On the one hand, there are real changes occasioned by the assertions of the author, usually to the effect that she believes some proposition, but sometimes also to the effect that she disbelieves some proposition or that she suspends judgement on it. In particular, the acceptance status of the premises and the conclusion of an argument need to be processed. On the other hand, purely hypothetical changes are necessary to check, for instance, whether accepting the premises would result in accepting the conclusion or whether this would happen if the conclusion were not believed to be true anyway. Such hypothetical changes are exclusively used to test for enthymematic validity. Only real changes enter into the evolving ascription of a belief state to the author. Employing hypothetical changes enables us to cover both arguments with conclusions that have already been accepted before, and arguments with rejected premises; that is, arguments that are presented "just for the sake of argument".

We have argued that various degrees of interpretive charity can be invested in the interpretation of an argument. This is one reason why there is no unique outcome of 
the interpretation process. Ultimately, the result of an interpreter's effort will depend on whether he judges it to be more likely that the author presents an argument that is not even enthymematically valid, or that he, the interpreter, needs to revise his current ascription of a belief state to her, the author. In the latter case, a revision of the previously ascribed belief state by a conditional can turn an argument that is prima facie invalid into a enthymematically valid one. However, this is very different from "completing" an enthymeme by adding a specific premise. And the interpreter needs judgement, not a formal theory, to decide whether this amount of charity makes sense.

Acknowledgments We would like to thank audiences in Constance, Lund, Prague, Regensburg, Salzburg and Stockholm, and in particular Gregor Betz, Ralf Busse, John Cantwell, Eduardo Fermé, Sven Ove Hansson, Eva-Maria Konrad, Christoph Lumer, Jaroslav Peregrin, Friedrich Reinmuth, Vladimir Svoboda as well as two anonymous referees of this journal for numerous critical comments on earlier versions of this paper. They have been very helpful.

\section{References}

Arieli, O., Denecker, M., \& Bruynooghe, M. (2007). Distance semantics for database repair. Annals of Mathematics and Artificial Intelligence, 50, 389-415.

Boutilier, C. (1996). Iterated revision and minimal change of conditional beliefs. Journal of Philosophical Logic, 25, 263-305.

Boutilier, C., \& Goldszmidt, M. (1995). On the revision of conditional belief sets. In G. Crocco, L. F. del Cerro, \& A. Herzig (Eds.), Conditionals. From philosophy to computer science (pp. 267-300). Oxford: Oxford University Press.

Brun, G. (2004). Die richtige Formel. Philosophische Probleme der logischen Formalisierung (2nd ed.). Frankfurt a. M.: Ontos.

Brun, G. (2008). Formalization and the objects of logic. Erkenntnis, 69, 1-30.

Brun, G., \& Hirsch Hadorn, G. (2009). Textanalyse in den Wissenschaften. Inhalte und Argumente analysieren und verstehen. Zürich: vdf.

Clark, H. H. (1996). Using language. Cambridge: Cambridge University Press.

Davidson, D. (1980). The logical form of action sentences. Criticism, comment, and defence. In Essays on actions and events (pp. 122-148). Oxford: Oxford University Press.

Davidson, D. (1984). Radical interpretation. In Inquiries into truth and interpretation (pp. 125-139). Oxford: Oxford University Press.

Ennis, R. H. (1982). Identifying implicit assumptions. Synthese, 51, 61-86.

Gärdenfors, P. (1979). Conditionals and changes of belief. In I. Niiniluoto \& R. Tuomela (Eds.), The logic and epistemology of scientific change. Acta Philosophica Fennica (Vol. 30, pp. 381-404). Amsterdam: North-Holland.

Gärdenfors, P. (1986). Belief revisions and the Ramsey test for conditionals. Philosophical Review, 95 , 81-93.

Gärdenfors, P. (1988). Knowledge in flux. Cambridge, MA: MIT Press.

Gärdenfors, P., \& Rott, H. (1995). Belief revision. In D. M. Gabbay, C. J. Hogger, \& J. A. Robinson (Eds.), Handbook of logic in artificial intelligence and logic programming. Vol. IV. Epistemic and temporal reasoning (pp. 35-132). Oxford: Oxford University Press.

Gerritsen, S. (2001). Unexpressed premises. In F. H. van Eemeren (Ed.), Crucial concepts in argumentation theory (pp. 51-79). Amsterdam: Amsterdam University Press.

Grandy, R. E. (1973). Reference, meaning, and belief. Journal of Philosophy, 70, 439-452.

Grice, H. P. (1975). Logic and conversation. In P. Cole \& J. L. Morgan (Eds.), Syntax and semantics. Vol. 3. Speech acts (pp. 41-58). New York: Academic Press. (Reprinted in Studies in the way of words (pp. 22-40). Cambridge, MA: Harvard University Press, 1989).

Hansson, S. O. (1992). Similarity semantics and minimal changes of belief. Erkenntnis, 37, 401-429.

Jacquette, D. (1996). Charity and the reiteration problem for enthymemes. Informal Logic, 18, 1-15.

Katsuno, H., \& Mendelzon, A. O. (1991). Propositional knowledge base revision and minimal change. Artificial Intelligence, 52, 263-294. 
Kern-Isberner, G. (1999). Postulates for conditional belief revision. In International joint conference on artificial intelligence (pp. 186-191).

Konieczny, S., \& Pino-Pérez, R. (2002). Merging information under constraints. A logical framework. Journal of Logic and Computation, 12, 773-808.

Lehmann, D., Magidor, M., \& Schlechta, K. (2001). Distance semantics for belief revision. Journal of Symbolic Logic, 66, 295-317.

Leitgeb, H. (2010). On the Ramsey test without triviality. Notre Dame Journal of Formal Logic, 51, 21-54.

Lindström, S., \& Rabinowicz, W. (1998). Conditionals and the Ramsey test. In D. M. Gabbay \& Ph. Smets (Eds.), Handbook of defeasible reasoning and uncertainty management systems. Vol. 3. Belief change (pp. 147-188). Dordrecht: Kluwer.

Lumer, Ch. (2005). The epistemological approach to argumentation. A map. Informal Logic, 25, 189-212.

Massey, G. J. (1975). Are there any good arguments that bad arguments are bad? Philosophy in Context, 4, 61-77.

Nayak, A., Pagnucco, M., Foo, N. Y., \& Peppas, P. (1996). Learning from conditionals. Judy Benjamin's other problems. In W. Wahlster (Ed.), ECAI 1996. Proceedings of the 12th European conference on artificial intelligence, August 11-16, 1996, Budapest, Hungary (pp. 75-79). New York: Wiley.

Nute, D., \& Cross, Ch B. (2001). Conditional logic. In D. M. Gabbay \& F. Guenthner (Eds.), Handbook of philosophical logic (2nd ed., Vol. 4, pp. 1-98). Dordrecht: Kluwer.

Oliver, J. W. (1967). Formal fallacies and other invalid arguments. Mind, 76, 463-478.

Paglieri, F., \& Castelfranchi, C. (2006). The Toulmin test. Framing argumentation within belief revision. In D. Hitchcock (Ed.), Arguing on the Toulmin model. New essays in argument analysis and evaluation (pp. 359-377). Dordrecht: Springer.

Paglieri, F., \& Woods, J. (2011). Enthymematic parsimony. Synthese, 178, 461-501.

Peppas, P., Foo, N., \& Nayak, A. (2000). Measuring similarity in belief revision. Journal of Logic and Computation, 10, 603-619.

Ramsey, F. P. (1931). General propositions and causality. In R. B. Braithwaite (Ed.), The foundations of mathematics and other logical essays (pp. 237-255). London: Routledge and Kegan Paul.

Rott, H. (2008). Belief revision. In J. Adler \& L. Rips (Eds.), Reasoning. Studies of human inference and its foundations (pp. 514-534). Cambridge: Cambridge University Press.

Rott, H. (2009). Shifting priorities: Simple representations for twenty-seven iterated theory change operators. In D. Makinson, J. Malinowski, \& H. Wansing (Eds.), Towards mathematical philosophy (pp. 269-296). Dordrecht: Springer.

Rott, H. (2011). Reapproaching Ramsey. Conditionals and iterated belief change in the spirit of AGM. Journal of Philosophical Logic, 40, 155-191.

Scholz, O. R. (2001). Verstehen und Rationalität. Untersuchungen zu den Grundlagen von Hermeneutik und Sprachphilosophie (2nd ed.). Frankfurt a. M.: Klostermann.

Sellars, W. (1953). Inference and meaning. Mind, 62, 313-338.

Spohn, W. (1988). Ordinal conditional functions. A dynamic theory of epistemic states. In W. L. Harper \& B. Skyrms (Eds.), Causation in decision, belief change, and statistics (Vol. 2, pp. 105-134). Dordrecht: Kluwer. (Reprinted in Spohn, W. (2009). Causation, coherence and concepts. A collection of essays (pp. 19-41). Dordrecht: Springer.

Spohn, W. (2012). The laws of belief. Ranking theory and its philosophical applications. Oxford: Oxford University Press.

Stalnaker, R. (1968). A theory of conditionals. In N. Rescher (Ed.), Studies in logical theory (APQ monograph series) (Vol. 2, pp. 98-112). Oxford: Blackwell. (Reprinted in Harper, W. L., Stalnaker, R., \& Pearce, G. (Eds.). (1980). Ifs. Conditionals, belief, decision, chance, and time (pp. 41-55). Dordrecht: Kluwer.

Stalnaker, R. (2002). Common ground. Linguistics and Philosophy, 25, 701-721.

Turing, A. M. (1950). Computing machinery and intelligence. Mind, 59, 433-460.

van Eemeren, F. H., \& Grootendorst, R. (1982). Unexpected [recte unexpressed] premisses. Part I. Journal of the American Forensic Association, 19, 97-106.

van Eemeren, F. H., \& Grootendorst, R. (2004). A systematic theory of argumentation. The pragmadialectical approach. Cambridge: Cambridge University Press.

van Rees, M. A. (2001). Argumentation interpretation and reconstruction. In F. H. van Eemeren (Ed.), Crucial concepts in argumentation theory (pp. 165-199). Amsterdam: Amsterdam University Press.

Walton, D. N., \& Macagno, F. (2009). Enthymemes, argumentation schemes and topics. Logique et Analyse, 205, 39-56. 